NASA Technical Memorandum 102867

\title{
Application of CFD to Sonic Boom Near and Mid Flow-Field Prediction
}

Samson H. Cheung, Thomas A. Edwards, and Scott L. Lawrence

(NASA-TM-1OPEST) APPLICATINN UF CFR TU

SONIC UOSM VEAR ANU MIU FLOW-FIEL? PQEOICTIUN (NASA) 130

CSCL OIA 
NASA Technical Memorandum 102867

\section{Application of CFD to Sonic Boom Near and Mid Flow-Field Prediction}

Samson H. Cheung, MCAT Institute, San Jose, California

Thomas A. Edwards and Scott L. Lawrence, Ames Research Center, Moffett Field, California

August 1990

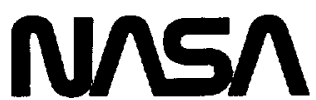

National Aeronautics and

Space Administration 



\title{
APPLICATION OF CFD TO SONIC BOOM NEAR AND MID FLOW-FIELD PREDICTION
}

\author{
Samson H. Cheung* \\ MCAT Institute, San Jose, California \\ Thomas A. Edwards ${ }^{* *}$ and Scou L. Lawrence ${ }^{\dagger}$ \\ NASA Ames Research Center, Moffet Field, California
}

\begin{abstract}
A three-dimensional parabolized Navier-Stokes (PNS) code has been used to calculate the supersonic overpressures from three different geometries at near- and mid-flow fields. Wind-tunnel data is used for code validation. Comparison of the computed results with different grid refinements is shown in this paper. It is observed that a large number of grid points is needed to resolve the tail shock/expansion fan interaction. Therefore, an adaptive grid approach is employed to calculate the flow field. The agreement between the numerical results and the windtunnel data confirms that computational fluid dynamics can be applied to the problem of sonic boom prediction.
\end{abstract}

\section{Introduction}

The sonic boom disturbance produced by current commercial supersonic transports is too disruptive for routine overland flight. Since sonic booms are environmentally objectionable, commercial supersonic flight over land is currently banned throughout most of the world. It is known that by redistributing the airplane lift distribution, the pressure pulse associated with the sonic boom can be alleviated. ${ }^{1}$ In order to design an appropriate aircraft configuration, sonic boom prediction must be accurate.

Different types of equations are needed for sonic boom prediction in the near-, mid- and far-fields. This is illustrated by the sketch in Figure 1. The near field includes the flow relatively close to the body, normally within a few characteristic lengths. In general, the detailed

\footnotetext{
"Research Scientist. Member AIAA.

-Assistant Chief. Applied Computational Fluids Branch. Member AIAA.

${ }^{\dagger}$ Research Scientist. Member AIAA.

Copyright 11990 by the American Institute of Aeronautics and Astronautics, Inc. No copyright is asserted in the United States under Title 17, U.S. Code. The U.S. Government has a royalty-free license to exercise all rights under the copyright claimed herein for Governmental purposes. All other rights are reserved by the copyright owner.
}

solution of the near-field flow equations is of interest in determining the surface pressure distribution for the calculation of forces on the body. However, when complex near-field solution techniques are employed to calculate the flow further from the body, such as for predicting the sonic boom, the numerical calculations become increasingly inaccurate and time consuming, and often unstable. The mid-field includes the flow from a few to perhaps a hundred characteristic lengths. Here the flow is entirely supersonic and can be considered inviscid. Shocks are sufficiently weak that the flow can be considered homentropic, however, the nonlinear behavior of the compressions and expansions produced by particular features of the body cannot be neglected. The far field is defined to be the flow far from the body, typically beyond several hundred to a thousand characteristic lengths. All intermediate shocks have decayed or coalesced with either the front or rear shock, and the overpressure signature has evolved into the classical $\mathrm{N}$-wave.

For more than two decades, many researchers have studied the nature of sonic booms. $1,2,3$ Wind-tunnel experiments ranging from simple geometries to complex aircraft configurations were conducted to determine the validity of sonic-boom-prediction theory and to seek lowboom configurations. 4,5 The basic sonic-boom theory originated in a classic paper by G. B. Whitham in 1952.6 Whitham's theory is a modification of linearized theory that permits the coalescence of disturbances into shocks for smooth bodies of revolution. Basic to its application is the formulation of the $F$-function which is related to the equivalent area distribution of the aircraft. For isentropic axisymmetric flow, the pressure disturbances at large distances from the aircraft can be expressed in terms of the $F$-function. An altemate formulation of the $F$-function that was derived by Lighthill ${ }^{7}$ for non-smooth bodies was shown to be better for sonic-boom prediction for smooth and non-smooth projectile shapes. Later, Walkden showed that the Whitham theory could be applied to winged bodies. 8

There are two main techniques for reducing sonic boom: (1) aerodynamic minimization and (2) exotic configurations. ${ }^{9}$ The first technique uses a calculation of an optimal flight altitude such that the $\mathrm{N}$-wave is minimized. The second uses a design of the aircraft so that the mid 
field pressure disturbance, for example a finite-rise shock, is retained on the ground before it turns into a noisy $\mathrm{N}$-wave. Recently, researchers have concentrated on the second technique. The $F$-function theory is used as a sonic-boom-prediction tool in the iterative loop of the low-boom aircraft design ${ }^{10}$ and wind-tunnel investigation. ${ }^{11}$ However, this linear theory fails in highly nonlinear flow, such as the flow at angle of attack, at higher Mach numbers (between 3 and 5), and flows involving caustics.

The present study considers the application of computational fluid dynamics (CFD) to the problem of sonicboom prediction, i.e., prediction of pressure disturbances at a designated distance from the aircraft. A threedimensional parabolized Navier-Stokes (PNS) code is applied to three sonic boom model problems. Calculated pressure signatures are compared with wind-tunnel data. An extrapolation method based on the $F$-function theory is employed to obtain the far-field overpressure signal from a near-field CFD solution.

\section{Numerical Scheme}

The PNS equations have been used for the efficient prediction of three-dimensional, steady, supersonic, viscous flow fields. The efficiency is achieved in both computer time and memory requirement because the equations can be solved using a space-marching technique rather than time-relaxation. Furthermore, since the PNS equations are valid in both the inviscid and viscous regions of the flow field, the interaction between these regions of the flow field is automatically taken into account. Recent design studies for high-speed civil transports have focused on Mach 2 to 3.2 at cruise. ${ }^{12}$ Since the design process is iterative, a fast prediction method is needed, therefore, the PNS approach is used in predicting the pressure disturbances.

Nevertheless, there are certain limitations associated with "parabolization" of the full Navier-Stokes equations. The streamwise viscous terms are neglected. The inviscid region of the flow must be supersonic and the streamwise velocity component must be positive everywhere. The last requirement excludes streamwise flow separation, but crossflow separation is permitted.

The solution method used in this study integrates the PNS equations using an implicit, approximately factored, finite-volume algorithm in which the crossflow inviscid fluxes are evaluated by Roe's flux-difference splitting scheme. ${ }^{13}$ The upwind algorithm is used to improve the resolution of shock waves over that obtained with conventional central differencing schemes. The resulting compuler code has been successfully applied to a number of hypersonic problems including flow past a circular cone $\mathrm{e}^{14}$ and turbulent flow past a generic all-body hypersonic vehicle. $^{15}$

The application of the PNS code in this paper is twofold. First, the near-field solution is obtained by applying the code to the flow immediately surrounding the body. This solution can then be transferred to codes based on $F$-function theory to extrapolate to the far field. Second, exploratory calculations using the PNS code to perform the extrapolations are undertaken. Since the current sonic boom extrapolation capabilities are generally limited to linear methods, which assume isentropic flow and small disturbances, significant errors may occur at intermediate distances, where nonlinear effects are still present. Modification of the PNS code may be able to provide a propagation capability that incorporates all nonlinear effects, can easily be extended to include atmospheric gradients and turbulence, and has both two-dimensional and three-dimensional extrapolation capabilities.

\section{Whitham $\boldsymbol{F}$-Function Theory}

A currently accepted method of obtaining a pressure signature from the $F$-function based on an area balancing technique was first introduced by Whitham in 1952. This extrapolation theory assumes isentropic axisymmetric flow and small pressure disturbances. ${ }^{16}$ The $F$-function represents a distribution of sources which causes the same disturbances as the aircraft at a given distance from the aircraft, given by

$$
F(y)=\int_{0}^{y} \frac{S^{\prime \prime}}{\sqrt{y-\xi}} \mathrm{d} \xi
$$

where $S^{\prime \prime}$ is the second derivative of equivalent area distribution and $y=x-\beta r_{0}, \beta=\sqrt{M_{\infty}^{2}-1}$. Here $x$ is the streamwise distance and $r_{0}$ is the distance normal to the body. For the case $\beta r_{0} / y$ " 1 the pressure and the characteristic curve $\left.x\right|_{c}$ can be expressed in terms of $F$-function

$$
\begin{aligned}
& \frac{\Delta p}{p_{\infty}}=\gamma M_{\infty}^{2} F(y) / \sqrt{2 \beta r_{0}} \\
& \left.x\right|_{c}=y+\beta r_{0}-\kappa \sqrt{r_{0} F(y)}
\end{aligned}
$$

where $\gamma=1.4$ for air and $\kappa=(\gamma+1) M_{\infty}^{4} / \sqrt{2} \beta^{3 / 2}$. Note that, without knowing $S^{\prime \prime}$, the pressure signature at an arbitrary distance $r_{1}$ can be obtained by providing an overpressure signature at the near-field location $r_{0}$. First , obtain the $F(y)$ from pressure measurements at $r_{0}$ 


$$
\begin{array}{r}
F(y)=\frac{\Delta p}{p_{\infty}} \sqrt{2 \beta r_{0}} /\left(\gamma M_{\infty}^{2}\right) \\
y=\left.x\right|_{c}-\beta r_{0}+k \sqrt{r_{0} F(y)}
\end{array}
$$

and then define $y_{t}$ as

$$
y_{t}=x-\beta r_{1}=y-\kappa \sqrt{r_{1} F(y)}
$$

where $r_{1}>r_{0}$ is the desired distance. Since the pressure signal propagates at the local speed of sound, and each point of the signal advances according to its amplitude, the signal is distorted and the $F$-function becomes multivalued as shown in Fig. 2. A new $F$-function at $r_{1}$ is obtained by placing discontinuities (shocks) in such a way that the equal-area rule is satisfied (see Fig. 2). This new $F$-function gives the overpressure signature at $r_{1}$ by

$$
\begin{aligned}
& \frac{\Delta p}{p_{\infty}}=\gamma M_{\infty}^{2} F(y) / \sqrt{2 \beta r_{1}} \\
& \left.x\right|_{c}=y_{t}+\beta r_{1}
\end{aligned}
$$

\section{Sonic Boom Prediction}

Three configurations, a cone-cylinder, a low-aspectratio rectangular wing, and a delta-wing body, were studied. These were simple geometries for which wind-tunnel data were available for comparison. All calculations were done at zero angle of attack $(\alpha=0)$.

\section{Cone-Cylinder}

The cone-cylinder shown in Fig. 3 had a cone angle of $6.48^{\circ}$. In this test case the free-stream Mach number was 1.68 . The experimental overpressure signature was measured at distances of 10 and 20 cone lengths $(H / L=$ 10 and 20) from the cone where $H / L$ is the height per body length.

The calculation of the near-field solution for the halfplane used a grid containing 21 grid lines in the circumferential direction and 140 in the normal direction. In the streamwise direction, the marching code took a step size of about $1 \%$ of the cone length. The overpressure calculation at 10 and 20 cone lengths used the propagation capability of the PNS code. The near-field computation was used as a starting solution for an axisymmetric calculation on a grid containing two grid lines in the circumferential direction and 340 in the normal (radial) direction. This PNS extrapolation required 4 to 6 minutes of CPU time on the Cray-YMP to obtain the pressure signature at 10 cone lengths.
The overpressure predictions at 10 and 20 cone lengths shown in Figs. $4 \mathrm{a}$ and $4 \mathrm{~b}$, respectively, confirm the propagation capabilities of the code. All the numerical solutions shown in this paper were shifted to align the computational and experimental signatures at $\Delta p / p_{\infty}=0$ in the expansion region. The abscissa, $\Delta x / L$, is the shifted distance divided by the characteristic length. Figure $4 \mathrm{a}$ indicates the differences between the solutions obtained using a fine grid and a coarse grid. The coarse grid contains 140 points in the normal direction. Also, the effects of the marching step size on the PNS solution are shown in Fig. 5. The larger step size is $2 \%$ of the cone length. The bow shock is correctly captured on the coarse grid, but in order to resolve the tail shock/expansion fan interaction, more grid points are needed. This test case is a good test for the marching code, since the small freestream Mach number and small cone angle result in very weak shocks, and a weak expansion fan. The test was run at constant total pressure and hence variable Reynolds number due to changing total temperature. Overpressure signals at 10 cone lengths with $\mathrm{Re}=5 \times 10^{4}$ and $\mathrm{Re}=$ $1 \times 10^{6}$ are presented in Fig. 6. The calculations were done by assuming an adiabatic body surface and a freestream temperature of $275^{\circ} \mathrm{K}$. It is observed that including viscosity does not produce significant differences from the Euler calculation.

\section{Low-Aspect-Ratio Wing}

The second configuration considered was the lowaspect-ratio wing shown in Fig. 7a. The free-stream Mach number for the wind-tunnel test was 2.01. A sample computational surface grid is shown in Fig. $7 \mathrm{~b}$. The quarterplane contains 90 points in the circumferential direction and 140 in the radial direction. The overpressure signal was obtained one chord length below the wing.

Figure 8 shows that both the viscous (dotted line) and the inviscid (solid line) results agree well with the experiment except that the location of the tail shock is slightly downstream of the measured location most likely because the sting of the wind-tunnel model was not accounted for in the calculations. The thickness of the sting is almost half the maximum wing thickness and it attaches at $x / c=$ 0.933. Thus, in the experiment, the tail shock formed well upstream of the trailing edge, whereas omitting the sting in the computational model produced a shock attached to the trailing edge of the wing. Also notice that the tail shock for the viscous calculation is slightly downstream of the inviscid shock location. Because the PNS assumptions eliminate upstream influence, the presence of a boundary layer effectively delays the formation of a tail shock at the wing trailing edge. Boundary layer displacement also causes the bow shock to be slightly upstream of the inviscid prediction. 
The inviscid calculation of the overpressure at one body length required two hours of CPU time. Because of the step size restriction associated with the viscous grid clustering, the viscous calculation required eight hours of CPU time. Subsequent grid resolution studies have indicated that acceptable results can be obtained on a grid with half as many grid points in the circumferential direction, so that the CPU time can be halved.

The overpressure signals at one body length obtained from the viscous and inviscid calculations were used as input to a quasi-linear extrapolation code based on the Whitham $F$-function theory. This extrapolation theory, which assumes isentropic flow and small pressure disturbances, gives the overpressure signatures at eight body lengths, as shown in Fig. 9. It is seen that the numerical predictions agree well with the wind-tunnel data. The axisymmetric PNS extrapolation to 8 body lengths, also shown in Fig. 9, predicted the shock points and the peaks correctly, although the gradient of the shock jump is smeared. Inadequate grid resolution near the shock caused excessive dissipation and smearing of the pressure gradient.

\section{Delta-Wing Body}

The third configuration considered was the deltawing body shown in Fig. 10a. The free-stream Mach number for the wind-tunnel test was 2.7. The computational grid contained 70 points in the circumferential direction and 140 in the normal direction. Due to quadrilateral symmetry, the computational grid modeled the quarter plane only. An example of the computational grid is shown in Fig. 10b. The overpressure signal was obtained at a distance of 3.1 body lengths. Viscous and inviscid near-field solutions were obtained using the PNS code for the region from the nose to approximately one body length downstream of the trailing edge. The Euler solution was computed with a step size of $0.06 \%$ to $0.18 \%$ of the body length and required about 45 minutes of CPU time. The viscous solution was obtained using a step size of $0.018 \%$ of the body length and a computational grid that contained 40 circumferential points. This calculation required about 86 minutes of $\mathrm{CPU}$ time on the Cray-YMP.

The Euler solution at the vertical plane of symmetry was used as a starting solution for the axisymmetric PNS extrapolation, with two lines in the circumferential direction and 400 in the radial direction, as was described for the cone-cylinder case. This extrapolation process, using a step size of 0.01 , required about 40 minutes of CPU time on the Cray-YMP to march 10 body lengths to obtain the overpressure signature. This calculation defined all the shock points correctly and captured the bow shock and the wing shock, but the tail shock/expansion fan interaction was poorly resolved because of a lack of grid points. A series of numerical experiments, as indicated in Fig. 11, showed that increasing the number of grid points improved the prediction of the tail shock/expansion fan interaction. The solutions did not match well downstream from the tail-shock because the wind-tunnel model's sting was not included in the calculations.

The number of grid points required in these calculations for accurate sonic boom prediction led to the consideration of an adaptive grid. An adaptive grid has recently been implemented in the PNS code with successful results in two-dimensional and axisymmetric calculations. ${ }^{17}$ In the present application, adaptive gridding was utilized only for the axisymmetric PNS extrapolation and, thus, grids were only adapted in the radial direction.

Both the viscous and inviscid 3-D solutions at the station one body length downstream of the trailing edge have been used as starting solutions for the PNS extrapolation code. Numerical experiments showed that acceptable results could be obtained from the adaptive grid procedure with 340 radial grid points and a step size of 0.01 . The PNS extrapolations of the inviscid and viscous solutions required approximately 60 and 80 minutes CPU time, respectively. In Fig. 12 the inviscid solution indicates slightly improved results using adaptive grids. Both the viscous and inviscid solutions from the adaptive grid agree fairly well with the wind-tunnel data and are shown in Fig. 13. The differences between the viscous and inviscid solutions are similar to those observed in the preceding case: the leading-edge shocks are displaced upstream, and the trailing-edge shocks downstream, by a viscous PNS calculation. The improved prediction of the shock peaks by the viscous solution was primarily due to the smaller spatial marching step size necessitated by the fine grid. Numerical experiments have shown that an inviscid calculation with a comparable step size yields essentially the same result.

The overpressure signals at $1,1.5$, and 2 body lengths obtained from the viscous PNS calculation followed by the PNS extrapolation were used as inputs to the quasilinear extrapolation code. The results at 3.1 body lengths are shown in Fig. 14. It is noted that an acceptable result can be obtained by extrapolating the CFD solution from as near as one body length. Thus, CFD can provide a near-field flow solution for a quasi-linear extrapolation code based on Whitham $F$-function theory.

\section{Concluding Remarks}

Accurate sonic boom prediction is needed in the development of next-generation supersonic transports. Existing theories based on linearized flow analysis cannot provide sufficient accuracy. This study has demonstrated that CFD can provide near-field now solutions accurate enough to be used for sonic boom prediction. 
Furthermore, these results have shown that CFD can be used to propagate the pressure disturbances to the midfield and provide accurate answers. Thus CFD can be used as a tool in the iterative loop of the low-boom aircraft design. While this is computationally expensive compared to the linear propagation techniques, the approach is more versatile and may prove useful when more detailed information is needed.

Including viscous effects for these cases had a negligible effect on the far-field pressure signature. However, the improved grid resolution required to obtained viscous solutions demonstrated the sensitivity of the far-field signature to the shock and expansion resolution near the body. To alleviate the computational expense of extremely fine grids, a solution-adaptive grid procedure was implemented which produced accurate solutions with half as many grid points.

\section{References}

${ }^{1}$ Hayes, W. D., "Similarity Rules for Nonlinear Acoustic Propaga ion Through a Caustic," NASA SP-180, 1969, pp. 165-171.

${ }^{2}$ Seebass, R., "Nonlinear Acoustic Behavior at a Caustic," NASA SP-225, 1971, p. 87.

${ }^{3}$ Ferri, Antonio, Ting, Lu, and Lo, T., "Nonlinear Sonic Boom Propagation Including the Asymmetric Effects," AIAA J., Vol. 15, No. 5, May 1977, pp. 653-658.

${ }^{4}$ Hunton, L., Hicks, R., and Mendoza, J., "Some Effects of Wing Planform on Sonic Boom," NASA TN-D-7160, 1973.

${ }^{5}$ Mendoza, J. and Hicks, R., "Further Studies of The Extrapolation of Near-Field Overpressure Data," NASA TM-X-2219, 1971.

${ }^{6}$ Whitham, G. B., "The flow Pattern of a Supersonic Projectile," Comm. Pure \& Appl. Math., Vol. V, No. 3, Aug. 1952, pp. 301-348.
${ }^{7}$ Lighthill, M. J., "Higher Approximation," in General Theory of High Speed Aerodynamics, Section E, Vol. VI of High Speed Aerodynamics and Propulsion, Princeton University Press, 1954, pp. 345-389.

${ }^{8}$ Walkden, F., "The Shock Pattern of a Wing-Body Combination, Far From the Flight Path," Aeronaut. Q., Vol. IX, Pt. 2, May 1958, pp. 164-194.

${ }^{9}$ Seebass, R. and George, A., "Sonic Boom Minimization," J. of Acoustical Society of America, Vol. 51, No. 2 (part 3), 1972.

10 Darden C., "Sonic-Boom Minimization With NoseBluntness Relaxation," NASA TP-1348, 1979.

${ }^{11}$ Mack, R., "Wind-Tunnel Investigation of the Validity of a Sonic-Boom-Minimization Concept," NASA TP-1421, 1979.

${ }^{12}$ Morris, C., Winston, M., and Morris, S., Jr., "Some Key Considerations for High-Speed Civil Transports," AIAA Paper 88-4466, Sept. 1988.

${ }^{13}$ Roe, P. L. "Approximate Reimann Solvers, Parameter Vectors, and Difference Schemes," J. Computational Physics, Vol.43, 1981, pp. 357-372.

${ }^{14}$ Lawrence, S., Chaussee, D., and Tannehill, J., "Application of an Upwind Algorithm to the 3-D Parabolized Navier-Stokes Equations," AIAA Paper 87-1112, June 1987.

${ }^{15}$ Lockman, W. K., Lawrence, S. L., and Cleary, J. W., "Surface Heat Transfer and Pitot-Pressure Survey Results for an All-Body Hypersonic Aircraft," Paper No. 6, 7th National Aero-Space Plane Technology Symposium, Oct. 1989.

${ }^{16}$ Gottlieb, J. and Ritzel, D., "Analytical Study of Sonic Boom From Supersonic Projectiles," Prog. Aerospace Sci., Vol. 25, 1988, pp. 131-188.

${ }^{17}$ Harvey, A., Acharya, S., Lawrence S., and Cheung, S. "Solution Adaptive Grid Procedure for an Upwinded Parabolized Flow Solver," AIAA Paper 90-1567, June 1990. 


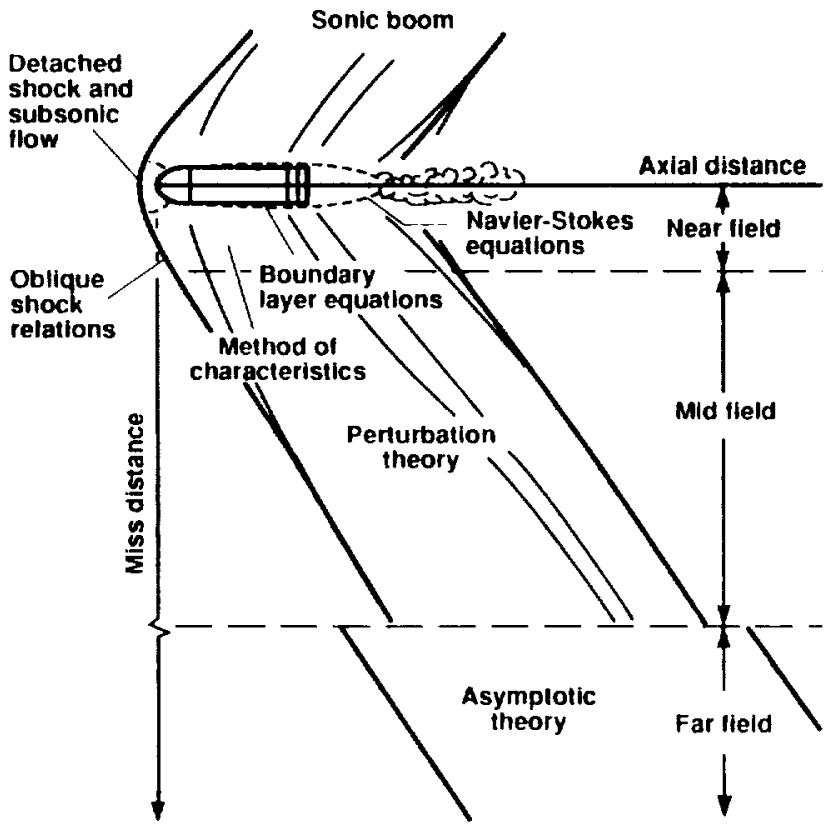

Fig. 1. Flow around a supersonic projectile showing the near-, mid-, and far-fields.

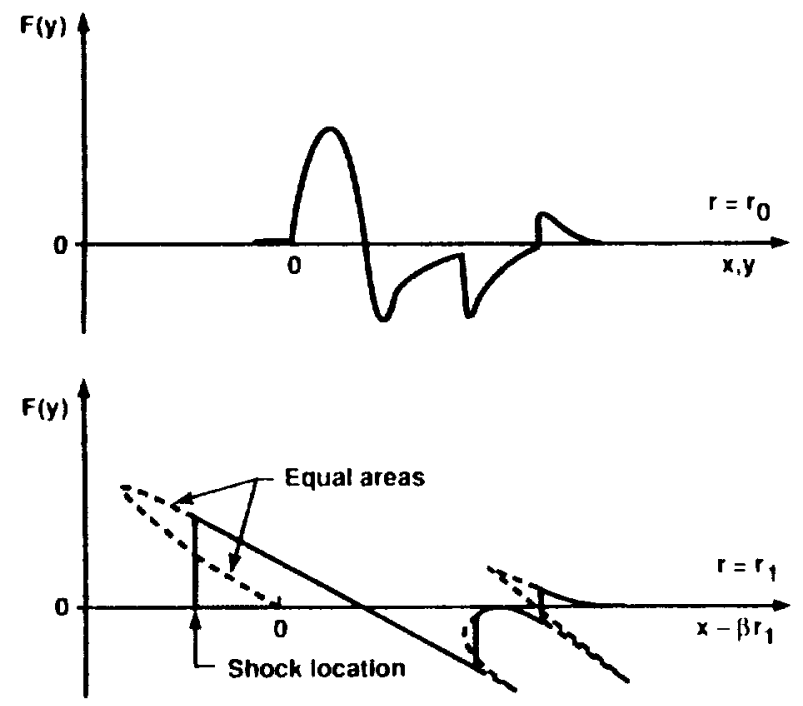

Fig. 2. The transposed $F$-function and the location of the shocks determined by the equal-area rule. The shaded areas are replaced by a discontinuity.

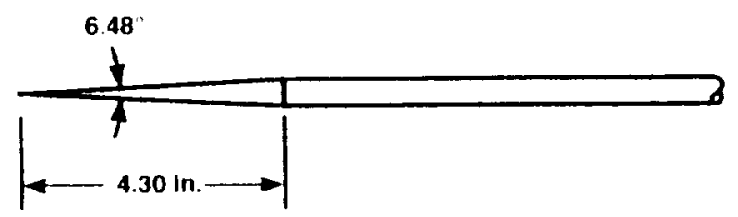

Fig. 3. Cone-cylinder model.
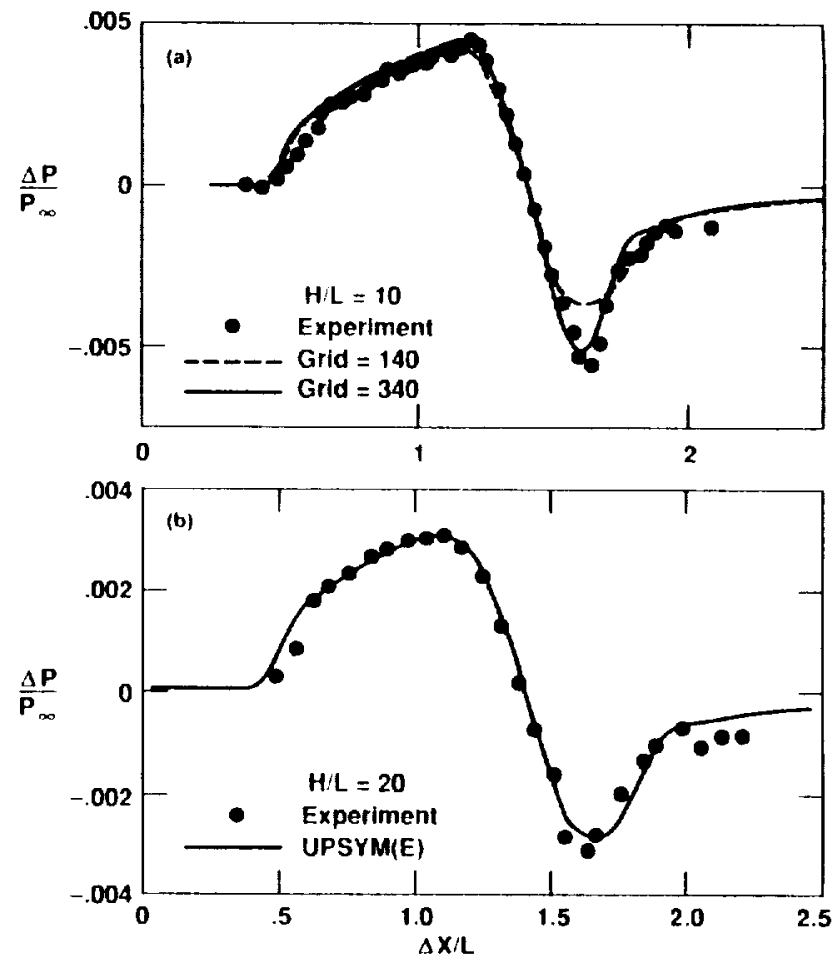

Fig. 4a) Variation of the overpressure signal with radial grid resolution. b) Overpressure signal at 20 cone lengths. A computational grid with 340 points in radial direction is used.

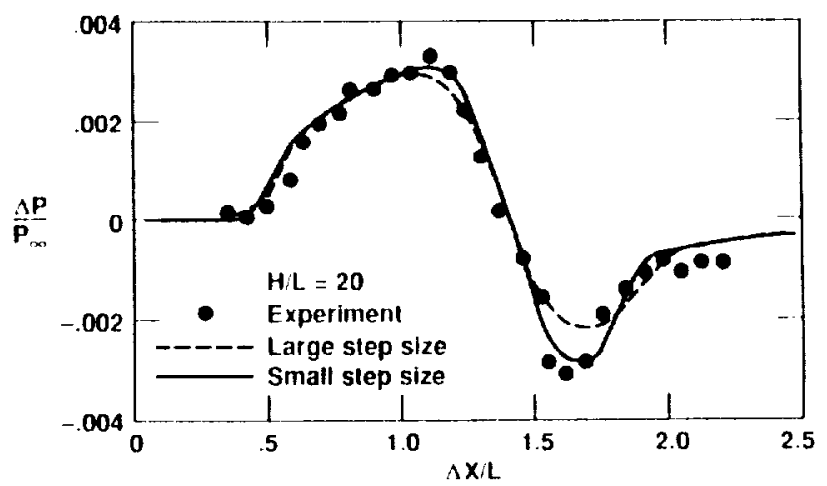

Fig. 5. Comparison of the overpressure signals at 20 cone lengths for different step sizes. 


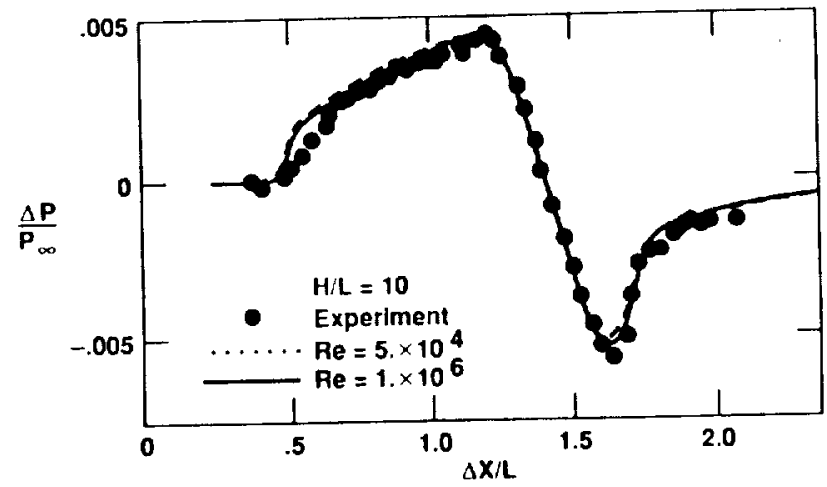

Fig. 6. Overpressure signals at 10 cone lengths with two different Reynolds numbers.
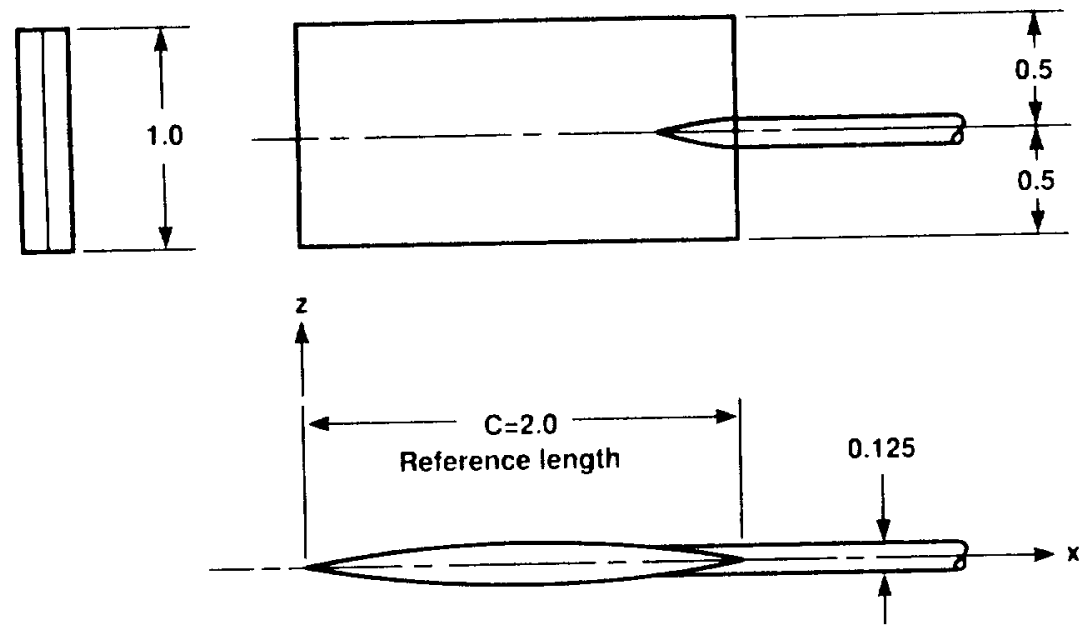

Thickness distribution is given by $z=-\frac{\pi}{12.5}\left(x-\frac{x^{2}}{2}\right)$

(a)

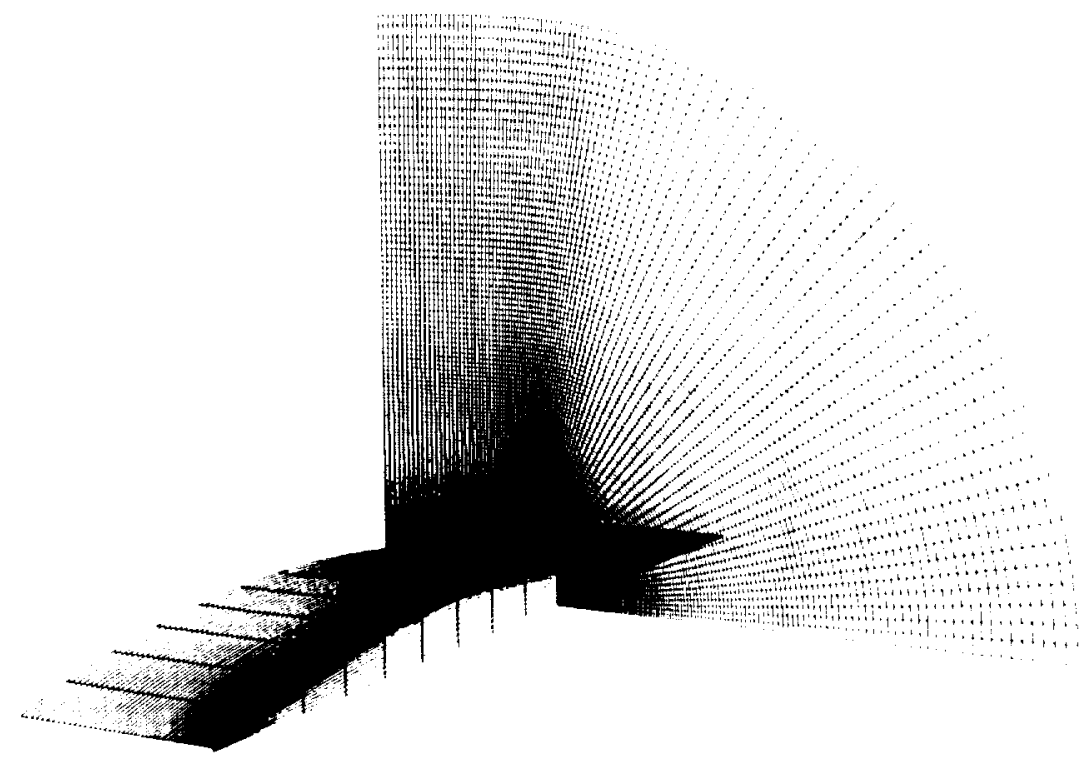

(b)

Fig. 7a) Geometry of the low-aspect-ratio wing. b) Computational grid of the low aspect ratio wing with 90 points in the circumferential direction and 140 in the normal direction. 


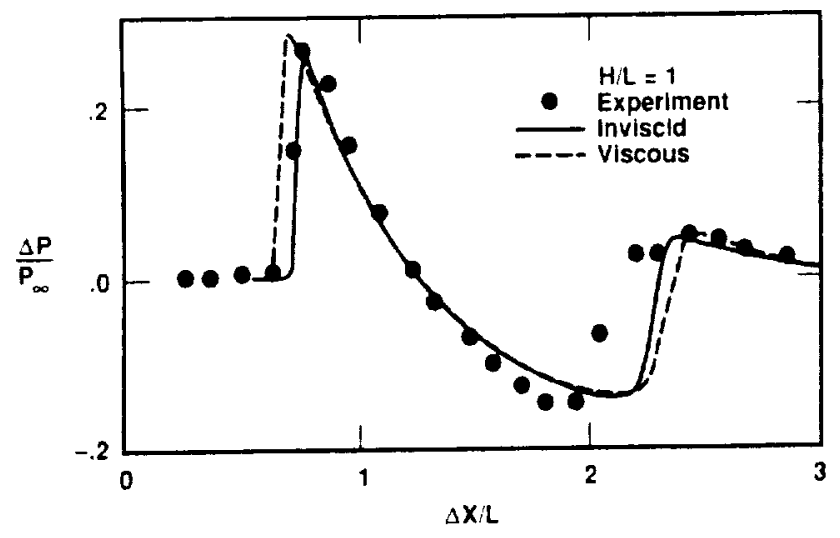

Fig. 8. Comparison of overpressure signals of viscous and inviscid calculations of the wing in the near-field.

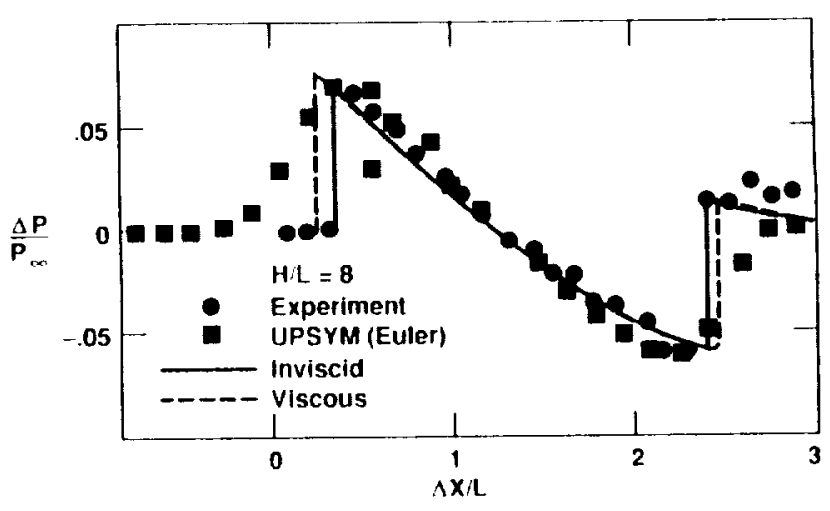

Fig. 9. Overpressure signal obtained at 8 chord lengths by extrapolation using the $F$-function theory from the signal at one chord length.

$$
r=0.540-0.011(x-7.01)^{2} \text { for all forebodies }
$$

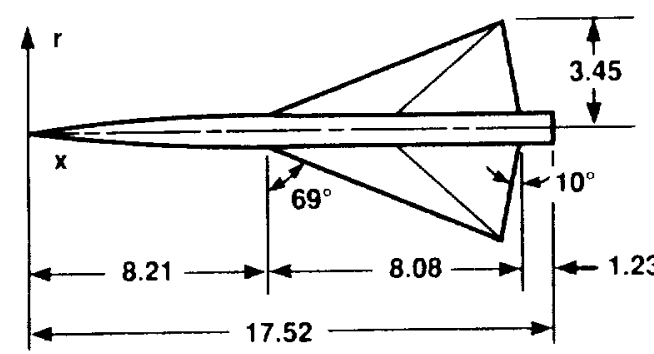

$$
t / c=0.05
$$

for all wing sections

(a)

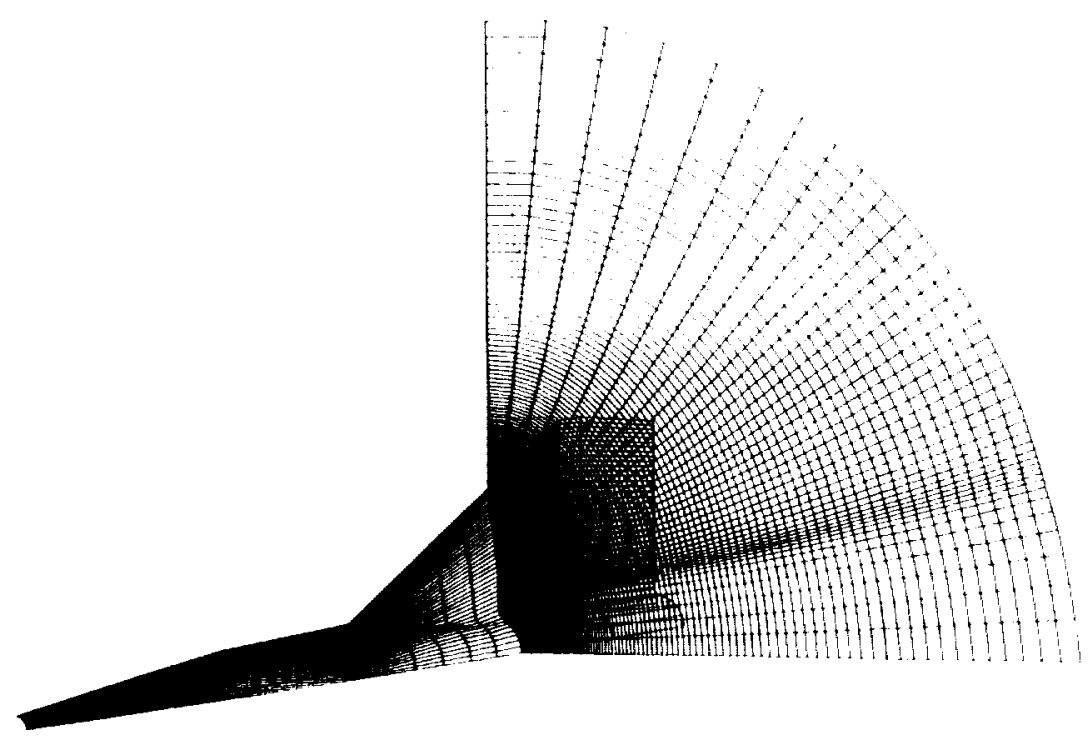

(b)

Fig. 10a) Geometry of the delta-wing body. b) Computational grid of the wing-body with 40 points in the circumferential direction and 140 in the normal direction. 


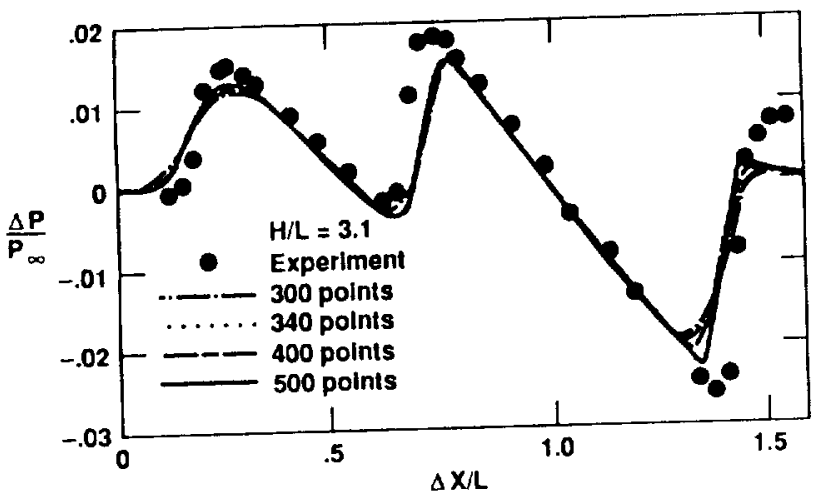

Fig. 11. Comparison of the overpressures of the wing body with grid refinement in the radial direction.

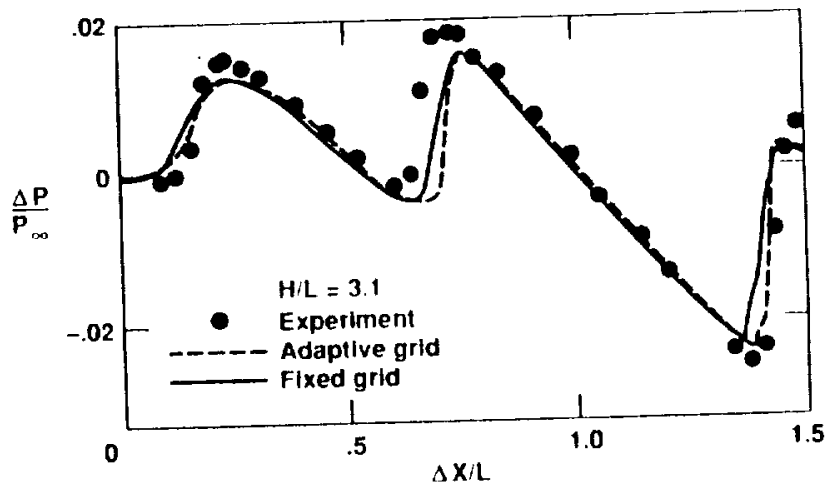

Fig. 12. Overpressures from the Euler calculations with fixed ( 540 points) and adaptive ( 340 points) grids.

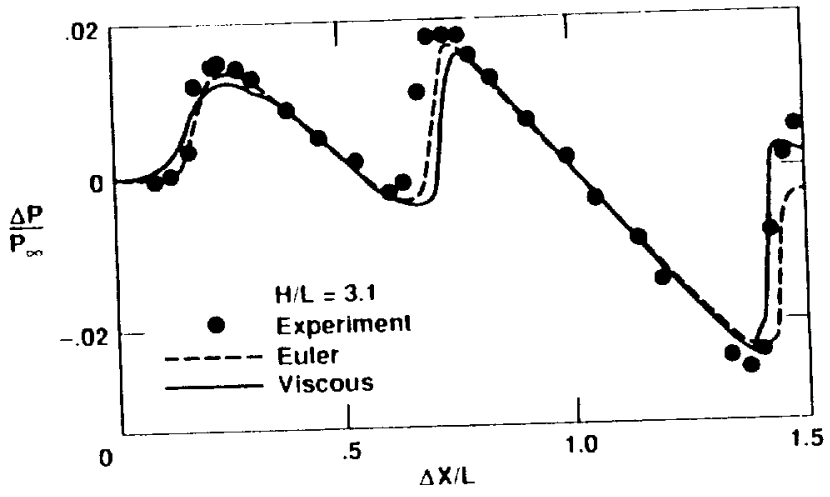

Fig. 13. Overpressures from viscous and inviscid calculations.

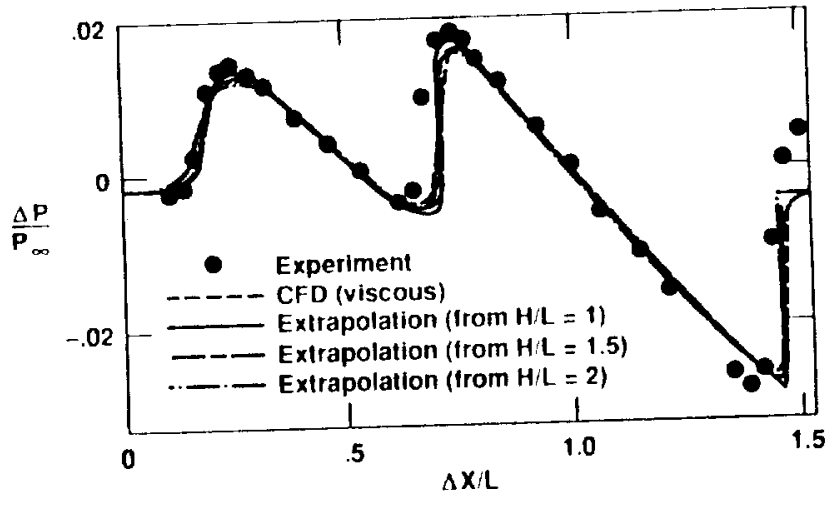

Fig. 14. Overpressure signal at 3.1 body lengths extrapolated from near distances. 


\begin{tabular}{|c|c|c|c|}
\hline NNSA & \multicolumn{3}{|c|}{ Report Documentation Page } \\
\hline $\begin{array}{l}\text { 1. Report No. } \\
\text { NASA TM-102867 }\end{array}$ & 2. Government Accession No. & \multicolumn{2}{|c|}{ 3. Recipient's Catalog No. } \\
\hline \multirow{2}{*}{\multicolumn{2}{|c|}{$\begin{array}{l}\text { 4. Tite and Subtite } \\
\text { Application of CFD to Sonic Boom Near and Mid Flow-Field } \\
\text { Prediction }\end{array}$}} & \multicolumn{2}{|c|}{$\begin{array}{l}\text { 5. Report Dale } \\
\text { August } 1990\end{array}$} \\
\hline & & \multicolumn{2}{|c|}{ 6. Performing Organization Code } \\
\hline \multirow{2}{*}{\multicolumn{2}{|c|}{$\begin{array}{l}\text { 7. Author(s) } \\
\text { Saǹson H. Cheung (MCAT Institute, San Jose, CA), } \\
\text { Thomas A. Edwards, and Scott L. Lawrence }\end{array}$}} & \multicolumn{2}{|c|}{$\begin{array}{l}\text { 8. Pertorming Organization Report No. } \\
\text { A-90246 }\end{array}$} \\
\hline & & \multicolumn{2}{|l|}{$\begin{array}{l}\text { 10. Work Unit No. } \\
537-03-21\end{array}$} \\
\hline \multicolumn{2}{|c|}{$\begin{array}{l}\text { 9. Pertorming Organization Name and Address } \\
\text { Ames Research Center } \\
\text { Moffett Field, CA } 94035-1000\end{array}$} & \multicolumn{2}{|c|}{ 11. Contract or Grant No. } \\
\hline \multirow{2}{*}{\multicolumn{2}{|c|}{$\begin{array}{l}\text { 12. Sponsoring Agency Name and Address } \\
\text { National Aeronautics and Space Administration } \\
\text { Washington, DC 20546-0001 }\end{array}$}} & \multicolumn{2}{|c|}{$\begin{array}{l}\text { 13. Type of Report and Period Covered } \\
\text { Technical Memorandum }\end{array}$} \\
\hline & & \multicolumn{2}{|c|}{ 14. Sponsoring Agency Code } \\
\hline \multicolumn{4}{|c|}{$\begin{array}{ll}\text { Point of Contact: Samson Cheung, Ames Research Center, MS } \\
\text { (415) 604-4462 or FTS 464-4462 }\end{array}$} \\
\hline \multicolumn{4}{|c|}{$\begin{array}{l}\text { 16. Abstract } \\
\text { A three-dimensional parabolized Navier-Stokes (PNS) code has been used to calculate the supersonic } \\
\text { overpressures from three different geometries at near- and mid-flow fields. Wind-tunnel data is used for } \\
\text { code validation. Comparison of the computed results with different grid refinements is shown in this paper. } \\
\text { It is observed that a large number of grid points is needed to resolve the tail shock/expansion fan interaction. } \\
\text { Therefore, an adaptive grid approach is employed to calculate the flow field. The agreement between the } \\
\text { numerical results and the wind-tunnel data confirms that computational fluid dynamics can be applied to } \\
\text { the problem of sonic boom prediction. }\end{array}$} \\
\hline \multicolumn{2}{|l|}{$\begin{array}{l}\text { 17. Key Words (Suggested by Author(s)) } \\
\text { Sonic boom } \\
\text { Prediction } \\
\text { CFD }\end{array}$} & $\begin{array}{l}\text { Unlimited } \\
\qquad \text { Subje }\end{array}$ & Category -02 \\
\hline $\begin{array}{l}\text { 19. Security Classit. (of this report) } \\
\text { Unclassified }\end{array}$ & $\begin{array}{l}\text { 20. Security Classit. (of this page) } \\
\text { Unclassified }\end{array}$ & $\begin{array}{l}\text { 21. No. of Pages } \\
10\end{array}$ & $\begin{aligned} \text { 22. Price } \\
\text { A02 }\end{aligned}$ \\
\hline
\end{tabular}



